\title{
La actividad agrícola en la secundaria básica cubana: análisis teórico
}

\section{Agricultural activity in cuban basic secondary school: theoretical analysis}

\author{
Bárbara Companioni Trujillo ${ }^{1}$ \\ bcompanioni@ucf.edu.cu \\ www.orcid.0000-0001-7029-4911
}

Fecha de recibo: 1/9/2019, Fecha de aceptación: 1/11/2019

\section{RESUMEN}

El trabajo, refleja un análisis teórico sobre la actividad, conociendo que es desde ella donde el hombre progresa y se desarrolla, teniendo en cuenta los diferentes tipos que existen, cada ser humano a lo largo de su vida suele y puede desarrollar diferentes tipos de actividades, práctica- cognitiva y valorativa, las cuales son inseparables. Es de ahí de donde se deriva entonces la actividad agrícola, como forma específica de trabajo, la cual posee una dimensión cognitiva que puede considerarse en este proceso, en el que se da la relación hombre naturaleza del conocimiento. También es intención de este trabajo el análisis desde el punto de vista psicológico, donde se explica, que la actividad de la persona desde los primeros años es regulada por la experiencia de la humanidad y las exigencias de la sociedad, por tanto, constituyen rasgos que la identifican, así como el interés de asumir la actividad agrícola como parte del proceso formativo de los alumnos de secundaria básica con una definición de la misma dirigida a esta enseñanza, trayendo como resultado además el desarrollo individual. Por lo anteriormente expuesto se distingue la estrecha relación que guardan los conceptos, de trabajo, habilidad, y trabajo socialmente útil los cuales se asumen para este estudio.

Palabras clave: Actividad, práctica, cognitiva, axiológica, agrícola, secundaria básica

\footnotetext{
${ }^{1}$ Universidad Carlos Rafael Rodríguez, Cienfuegos, Cuba.
}

Revista científica Ciencia y Tecnología Vol 20 No 25 págs. 110-117

http://cienciaytecnologia.uteg.edu.ec 


\begin{abstract}
The work reflects a theoretical analysis of the activity, knowing that it is from there that man progresses and develops, taking into account the different types that exist, each human being throughout his life usually and can develop different types of activities, practical-cognitive and evaluative, which are inseparable. It is from there that agricultural activity is derived, as a specific form of work, which has a cognitive dimension that can be considered in this process, in which the man-nature relationship of knowledge occurs. The intention of this work is also the analysis from the psychological point of view, where it is explained that the activity of the person from the first years is regulated by the experience of humanity and the demands of society, therefore, they are features that they identify it, as well as the interest in assuming the agricultural activity as part of the formative process of the students of basic secondary school with a definition of the same directed to this teaching, also resulting in individual development. For the foregoing, the close relationship between the concepts of work, ability, and socially useful work which are assumed for this study is distinguished.
\end{abstract}

Keywords: Activity, practice, cognitive, axiological, agricultural, basic secondary.

\title{
Introducción
}

La secundaria básica cubana de hoy está sometida a un cambio trascendental, que es expresión genuina de las transformaciones educativas que se están llevando a cabo en el marco de la tercera Revolución en materia de educación. Dichas transformaciones sustentan la necesidad de formar integralmente a los alumnos, lo cual significa la organización coherente y sistemática de las influencias educativas orientadas al desarrollo de hábitos, habilidades y valores acorde al momento histórico social que vive hoy la sociedad cubana.

Si bien la formación de una cultura general e integral se basa en la estrecha relación que existe entre la educación, la formación y el desarrollo, dicho objetivo se logra mediante la transmisión de conocimientos básicos de las distintas disciplinas, la formación de sentimientos y convicciones, la educación estética y la cultura física, el conocimiento elemental de los principios de la técnica relacionados con la actividad productiva, así como, el desarrollo de capacidades, habilidades y hábitos que les permitan a los alumnos incorporarse a la vida laboral, con un adecuado nivel de preparación, o realizar otros estudios, es por ello que como divisa fundamental se hace necesario la formación de los estudiantes desde el desarrollo de la actividad propia de los hombres en la que genera bienestar y desarrollo en todos los sentidos de ahí que se analice en este trabajo la actividad como base fundamental para este desarrollo. 
No. 25, 31 de enero de 2020

ISSN impreso: 1390 - 6321

ISSN online: 2661 - 6734

\section{Materiales y Métodos}

El método bibliográfico ha sido utilizado como parte del análisis que en este articulo tiene lugar, las diversas fuentes utilizadas aparecen referenciadas a lo largo del estudio y forman parte de las referencias que al final del artículo se ubican.

\section{Resultados y Discusión}

La actividad, como forma de existencia, desarrollo y transformación de la realidad social, penetra todas las facetas del quehacer humano, y en este sentido, tiene una connotación filosófica. En general, se ha definido como forma, específicamente humana, de relación activa hacia el mundo circundante, cuyo contenido es su cambio y transformación racional. Marx planteó como fundamento de la relación hombrenaturaleza, que la práctica social humana es capaz de transformar y reelaborar a la naturaleza y progresivamente hacerla su objeto, para su criterio... "al crear un mundo objetivo con su actividad práctica, al elaborar la naturaleza inorgánica, el hombre prueba ser un ser consciente (78).

Martínez (2017) trata el tema sobre los cotos educativos. Por otro lado, en específico, la actividad práctica, asumiendo lo planteado por el Dr. R. Pupo, es la "... categoría filosófica que designa la actividad material, adecuada a fines, es decir, una esencial relación sujeto - objeto donde lo ideal y lo material se convierten recíprocamente" ( $R$. Pupo, p. 75).

Los tres elementos: práctica- conocimiento - valor, constituyen momentos de la actividad inseparables, son aspectos de un todo único que solo se aíslan mediante la abstracción. De este modo, los conocimientos y valores son el resultado de la actividad del hombre. Este conoce y llega a una valoración de la realidad porque actúa prácticamente:

En el proceso de su actividad los hombres producen, ante todo, las condiciones materiales de su existencia: medios de vida y medios de trabajo. Simultáneamente... producen las formas de relaciones correspondientes a estas condiciones: económicas, políticas, etc. Conjuntamente con la producción de la vida material, los hombres producen su conciencia: las ideas, representaciones, conocimientos. En otras palabras, en el proceso de producción social los hombres crean toda la conjunción de premisas materiales y espirituales de su existencia en la sociedad y por consiguiente su existencia social (1989, p. 115).

La práctica social y específicamente, su forma determinada el trabajo, como forma especial y humana de mediar la relación sujeto- objeto, no conduce solo a la creación de objetos necesarios para la vida, sino además, deviene acto de objetivación de fines e ideas en la realidad; el trabajo es el núcleo de la práctica. 
En la actividad laboral, el hombre, humaniza la naturaleza creando objetos que satisfacen sus necesidades y al mismo tiempo, materializa sus propios proyectos ideales, engendrados por las necesidades prácticas de su ser esencial. Luego, la actividad práctica deviene punto de partida en esta relación; mientras, la cognoscitiva y valorativa, constituyen desprendimientos de aquella y sus resultados (conocimientos y valores).

La actividad cognoscitiva es una forma especial de la actividad espiritual del hombre condicionada por la práctica, donde se refleja la realidad y esta se reproduce en forma de conocimiento expresada en principios, leyes, categorías, hipótesis, teorías etc. El conocimiento media toda la actividad humana, incluyendo su fundamento sustancial: la práctica. "El conocimiento, es el reflejo de la naturaleza por el hombre. Pero no es un reflejo simple, inmediato, completo, sino el proceso de una serie de abstracciones, la formación y el desarrollo de conceptos, leyes, etc. Y estos conceptos, leyes etc., abarcan condicional, aproximadamente, el carácter universal, regido por leyes, de la naturaleza en eterno desarrollo y movimiento" (pág. 176).

En tanto, la actividad valorativa o axiológica es el modo en que existen las necesidades e intereses del hombre, la actividad valorativa, engendrada en la práctica social y alumbrada por el conocimiento, sirve de vínculo a la relación conocimiento-práctica, el hombre evalúa la realidad en función de sus necesidades y en la actividad práctica transformadora elabora fines para su satisfacción. En su obra "El Capital." Carlos Marx planteó cómo "la propia posibilidad de satisfacer una necesidad se genera en la relación práctico valorativa y se realza en ella, pues en la actividad transformadora el hombre asimila bajo una forma útil para su propia vida, las materias que la naturaleza le brinda" (p. 139). Por supuesto esto se cumple en todo tipo de actividad y de hecho en la actividad agrícola.

La actividad agrícola, como forma específica de trabajo, posee una dimensión cognitiva que puede considerarse básica. A través de ella se manifiesta la interacción dialéctica del hombre con la naturaleza y el resultado que de él se espera, además de obtener, determinado conocimiento del objeto en transformación: Durante este proceso, se ejecutan diversas acciones que no se dan de forma aislada sino en sistema y que constituyen la esencia de un proceso transformador de base científica, por tanto, se deben precisar los conocimientos elementales (especializados) para poder desarrollar la actividad agrícola.

Por lo general, el conocimiento asociado a este tipo de actividad cursa siempre desde la empíria Muchos obreros, campesinos, trabajadores de la agricultura aprenden su oficio por trasmisión de la experiencia acumulada de generación en generación), pero en la actualidad existe un desarrollo tecnológico y científico que le sirve de base para la aplicación extensiva de esta actividad y para el perfeccionamiento del proceso y los resultados. Si bien es cierto que el conocimiento se funda y está determinado por ella, esta resulta estéril al margen del conocimiento. Se entiende, entonces, que la dimensión cognitiva de la actividad agrícola descansa en la relación entre conocimiento y práctica, ellas están estrechamente vinculadas y se interpenetran recíprocamente.

\section{3}

Companioni. La actividad agrícola en la secundaria básica cubana: análisis teórico 
La actividad valorativa se asume como característica de la agrícola, en la medida que a través de ella se consigue desarrollar la capacidad o posibilidad de satisfacer determinadas necesidades humanas y de servir a la práctica social como base para su subsistencia. Determina por tanto la significación que el hombre y la sociedad le atribuyen al trabajo con la tierra; la cual está condicionada, no tanto por las propiedades naturales del proceso sino por el sistema de relaciones y por el propio resultado de esa interacción.

Se debe tener en cuenta que el aspecto valorativo de la actividad agrícola se expresa, también, en la capacidad creadora del hombre en todas sus manifestaciones, lo cual se inicia por la creación de los medios, procedimientos, técnicas y sistemas que movido por las necesidades prácticas es capaz de resolver. A esto se agrega que en la interrelación entre práctica y valoración juega un papel especial el proceso del propio conocimiento, mediando la unidad de la conciencia y la práctica o sea la relación entre los fines cognoscitivos y prácticos de la actividad humana.

Las relaciones axiológicas y gnoseológicas se interpretan recíprocamente en el marco de la práctica social, y el resultado final se da como síntesis de los componentes cognoscitivos, valorativos y prácticos de las acciones que concreta su tipología. El proceso de trabajo y de producción se asume como apropiación práctica espiritual de la realidad y reproducción de las relaciones sociales. Esta especificidad de éste tipo de actividad permanece invariable también en toda actividad humana, ya que el hombre asume la naturaleza, no-solo como objeto del conocimiento, sino como objeto de valoración y transformación.

Desde el punto de vista psicológico, se entiende que la actividad de la persona (desde los primeros años) es regulada por la experiencia de la humanidad y las exigencias de la sociedad, por tanto, constituyen rasgos que la identifican. El contenido de cualquier actividad no es determinado por la finalidad de ésta, los motivos que generan la acción del hombre sobre la tierra, aun cuando divergen en su fin inmediato, expresan la certeza histórico social que de ella emerge la conciencia de productor para satisfacer determinada necesidad y es dirigida con un fin consiente.

La actividad agrícola es una forma de relación viva ya que el hombre actúa sobre la naturaleza, las cosas y otras personas, establece un vínculo real entre este y el mundo que los rodea. En este tipo de actividad, tiene lugar la formación y el desarrollo de su conciencia como ser social productivo. Es ésta la contribución básica de la actividad agrícola en la formación y desarrollo de la personalidad del hombre.

En este marco se justifica, entonces, el interés de este trabajo de asumir la actividad agrícola como parte del proceso formativo de los alumnos en secundaria básica. 
Si bien toda actividad implica aprendizaje, en éste tipo específico, este se alcanza no solo en la práctica que se considera fundamental, sino también a través de las acciones pedagógicas organizadas y dirigidas con el objetivo de asimilar determinados conocimientos, hábitos y destrezas que preparen para este tipo de trabajo. Esto implica desarrollar un proceso pedagógico orientado a garantizar un comportamiento genérico específico del hombre relacionado con su supervivencia, el dominio de las fuerzas de la naturaleza y la socialización de nuestra intervención a través del trabajo.

Estimular, por tanto, el conocimiento que refleja la evolución del carácter polifacético del hombre y su creatividad en el intercambio con la naturaleza, garantiza que pueda satisfacer sus necesidades.

Se reconoce, entonces, que la actividad agrícola pueda convertirse en objetivo de formación en la medida que implique desarrollo de la conciencia de las personas, además, es contenido del aprendizaje cuando a través de esta se pueda asimilar determinados conocimientos, hábitos y destreza. Es método de enseñanza y educación, en tanto prepara al alumno para el trabajo garantizando su supervivencia; cursando además como medio, en tanto el ser humano, favorece, regula y controla su relación con la naturaleza, con sus actos, y contribuye a la formación de su personalidad.

El trabajo de los alumnos de secundaria básica debe corresponderse, en plena medida, con las características y demandas de esta edad. Cuando la situación se expresa contraria, aparece la pereza, la evasión de sus deberes laborales, la actividad negligente, los cuales son motivos de una educación incorrecta. Por tanto, al organizar y dirigir las actividades agrícolas debe proponerse el enriquecimiento de los conocimientos técnicos del alumno. Se debe lograr que él sienta el trabajo y le sirva como una nueva fuente de información, a través del cual pueda adquirir nuevos conocimientos y habilidades prácticas. Específicamente, el trabajo agrícola permite formar en ellos los hábitos de planificación y organización del trabajo; esto es válido para todas las actividades que se realizan con fines instructivos y formativos en la escuela, hogares y en todas las actividades socialmente útiles, en sus diversos aspectos y formas.

Por lo anteriormente expuesto vemos la estrecha relación que guardan los conceptos, de trabajo, habilidad, y trabajo socialmente útil los cuales se definen para este estudio como:

Trabajo: Es una actividad que, dado, sus rasgos característicos identifican a los hombres como ser social. Es un proceso de intercambio entre el hombre y la naturaleza, donde aquel transformando a ésta se cambia, se perfecciona a sí mismo y se relaciona con otros hombres 2000, p. 11). 
Habilidad: Es el dominio de un sistema complejo de acciones, necesarias para la regulación y realización conveniente de la actividad.

Está relacionado con el saber hacer (Menacho y Rojas, 2018). Las habilidades se adquieren y desarrollan en la actividad práctica, las cuales permiten que la información se convierta en un conocimiento verdadero, al mismo tiempo, que constituyen una expresión del conocimiento y en qué o hasta dónde pueden llegar los alumnos.

Trabajo socialmente útil: Es un tipo de trabajo dirigido a resolver problemas de la escuela y la comunidad en tareas concretas que van desde limpieza, ordenamiento y embellecimiento, hasta trabajo en el jardín, en el huerto, etc. (2000).

Para organizar las actividades agrícolas, la escuela y el profesor, en particular, deben apoyarse en la inspiración que caracteriza el descubrimiento de lo nuevo, matizada emocionalmente por la participación activa en la vida del colectivo y debe propiciar que el alumno acumule experiencias en las relaciones colectivistas y desarrolle el sentido del deber y la responsabilidad, así como, el ejercicio de ayuda mutua, solidaridad y la costumbre de supeditar, siempre que sea necesario, sus intereses en beneficio del colectivo. Mediante la actividad agrícola, la personalidad del alumno se irá formando dependiendo de cuál sea la experiencia que obtenga en ella: sea ésta cognoscitiva, práctica o valorativa y, por tanto, de su manera de actuar durante la ejecución de ésta.

Sin embargo, es la especificidad de la actividad agrícola, su ajuste al contexto escolar, la concepción didáctica en que se establece la relación entre los componentes del proceso, lo que permite concretar en la escuela el alcance formativo de ésta. Las escuelas ubicadas en el campo o con parcelas para la actividad agrícola tradicional, encuentran un espacio idóneo para realizar la experiencia que durante años ha desarrollado el país, pero las escuelas secundarias básicas urbanas se han orientado hacia una reconceptualización general del currículum que debe rescatar ciertas tradiciones y fortalecer otras nuevas.

Para este estudio se define a la actividad agrícola urbana en la secundaria básica como "toda actividad que desarrollan los alumnos para apropiarse de contenidos relacionados con la agricultura urbana en dos subprogramas fundamentales (hortalizas, condimentos frescos y plantas medicinales) a través de diferentes alternativas para resolver sus necesidades culturales.

Se entiende por necesidades culturales a todo lo que el hombre necesita dominar en el orden del conocimiento, de la práctica, de los valores, de forma general, que le son imprescindibles para vivir, como es por ejemplo alimentarse y hacerlo de forma adecuada. 
De ello se encarga la escuela, para lo cual se definen objetivos formativos que responden a los fines del ciclo escolar, pero sobre todo al proceso de formación de la personalidad.

\section{Conclusiones}

Sin lugar a dudas la actividad es fundamental para el desarrollo de cualquier individuo, importante en la educación básica por ser esta una edad importante en el desarrollo de una persona.

La actividad agrícola permite el desarrollo cultural y personal de un individuo, entiéndase por el dominio del conocimiento, la práctica, los valores, de forma general, que le son imprescindibles para vivir, como es por ejemplo alimentarse y hacerlo de forma adecuada.

\section{Referencias}

Cerezal Mezquita, J. (2000). El desarrollo de la concepción de la enseñanza de la Educación Laboral en la secundaria básica cubana a partir de 1975/ Julio Cerezal Mezquita. Tesis Doctoral en Ciencias Pedagógicas.

Companioni Turiño, B. Ponencia presentada al $V$ taller de Educación Laboral en Santiago de Cuba. El área de agricultura en la educación laboral en la secundaria básica en transformación y su contribución a la formación de valores morales en los alumnos.

Martínez, J. D. L., \& Banet, L. L. (2017). Cotos escolares: espacios educativos para la enseñanza de las ciencias en la escuela española del siglo XX. Bordón. Revista de pedagogía, 69(3), 161-174.

Martín Cala, M. (2012). Desde el problema científico hasta la introducción de los resultados en la práctica educativa EduSol, vol. 12, núm. 40, julio-septiembre, 2012, pp. 53-65 Centro Universitario de Guantánamo Guantánamo, Cuba

Marx, Carlos: Manuscritos económicos y filosóficos de 1844. La Habana: Editorial Progreso, 1980.

Marx, Carlos. El Capital. La Habana: Editorial Progreso, 1980.

Menacho, T., \& Rojas, M. (2018). Aula taller en el desarrollo de habilidades y capacidades profesionales en el curso de extensión agrícola del X ciclo Facultad Zootecnia de la Universidad Nacional Agraria de la Selva-Tingo María. RevIA, 3(1).

R.Pupo: "La actividad como relación sujeto objeto". Mezhuiev: La cultura y la Historia. La Habana: Editorial Progreso, 1980.

V. I. Lenin. (1972). Cuadernos Filosóficos. La Habana: Cartago.

Companioni. La actividad agrícola en la secundaria básica cubana: análisis teórico 